\title{
Improving Laser Ablation of Zirconia by Liquid Films: Multiple Influence of Liquids on Surface Machining and Nanoparticle Generation
}

\author{
Niko BÄRSCH ${ }^{* 1}$, Antonietta GATTI ${ }^{* 2}$, and Stephan BARCIKOWSKI ${ }^{* 1}$ \\ ${ }^{* 1}$ Laser Zentrum Hannover e.V., Hollerithallee 8, 30419 Hannover, Germany \\ E-mail:n.baersch@lzh.de \\ *2 Università di Modena e Reggio Emilia, via Campi 213 /A, 41100 Modena, Italy
}

\begin{abstract}
We have analyzed sample surfaces and particles generated during laser processing of tetragonal zirconia, considering ablation in the gas phase and in liquids using different energies and pulse durations. Remarkably, a liquid layer does not only allow collecting nanoparticulate matter and improving the quality of micromachining, but can also enhance material ablation rates.
\end{abstract}

DOI: $10.2961 / \mathrm{jlmn} .2009 .01 .0013$

Keywords: ceramic, zirconia, femtosecond laser ablation, micromachining, nanoparticles

\section{Introduction}

Laser ablation of tetragonal zirconia, a very hard ceramic widely-used in medical technology [1, 2], is the key to highly interesting new applications. On the one hand, short-pulsed laser structuring is the only wear-free method to shape fully sintered substrates, for instance to produce dental crowns [3]. On the other hand, particles that are laser-ablated from the surface of zirconia substrates can be embedded into other products to increase their surface strength. This laser-based nanotechnology approach provides the best results if ablated particles have specific characteristics (such as small size and good dispersion) and if a long-term fixation between such particles and the product is achieved [4]. The investigations show how zirconia ablation by femtosecond laser pulses affects micromachining quality and efficiency on the one hand and the amount and characteristics of generated nanoparticles on the other hand, and how ablation through liquid films can combine both aims.

\section{Motivation for fs laser machining of Y-TZP}

Zirconia with a tetragonal crystal structure is usually sintered from yttria-stabilized tetragonal zirconia polycrystal (Y-TZP) by hot isostatic pressing. It combines superior characteristics with regard to biocompatibility, mechanical durability, and optical appearance. However, its mechanical strength also bears problems for traditional processing.

The material properties of Y-TZP as a material for surgery implants are standardized, including test methods for microstructure and flexural strength [2]. On this basis, the flexural strength of typical sintered Y-TZP has been determined to between 900 and $1000 \mathrm{MPa}$ and the Weibull parameter to 10.7 to 14.9 [5], which means a toughness and reliability far beyond those of conventional surgical ceramics. The main reason for these mechanical advantages of YTZP ceramics is their good resistance against crack propagation due to a crystal transformation effect. Shear stress in the ceramic particles resulting from a beginning crack causes a transformation from the tetragonal to the mono- clinic phase. As this transformation leads to a volume expansion by 3-5\%, an internal stress increases the energy threshold for a further growth of the crack and thus inhibits a further crack opening $[1,6]$.

Commercial processing stations for ceramic frames meanwhile offer Computer-aided manufacturing (CADCAM) processes including the complete milling of presintered or fully-sintered Y-TZP blocks. However, when processing fully-sintered blocks, milling takes several hours and involves strong tool wear. Investigations have also indicated that an inner surface grinding does not only lead to a higher roughness at the produced inner surfaces, but significantly reduces the strength and reliability of the samples in comparison to lapped control samples [7].

Laser ablation processes, on the other hand, are contactless and wear-free. Ultrashort-pulsed lasers are an ideal tool for this task. They ablate Y-TZP in spite of its low absorption for most laser wavelengths, as the large intensities of focused fs laser pulses initiate the generation of free electrons at the ceramic surface. They ablate very precisely and avoid thermal effects that could otherwise cause phase transformations and reduce the sample strength. However, as opposed to the processing of metals, debris re-deposition can be a quality issue for laser machining of zirconia: for certain processing parameters, ablated material adheres tightly to the sample surface, constricting the ablation process and reducing the quality of results.

\section{Particulate ablation and re-deposition}

In the course of former investigations on fs laser ablation of Y-TZP in air, particulate emission from the processed surface has been analyzed using an electrical Coulomb low-pressure impactor, detecting the impaction of particles on twelve stages and converting measured currents to total numbers of particles of the respective size range. Fig. 1 shows the resulting particle size distribution for a typical ablation in air. During all measurements, the majority of particles in the fume were clearly in the nanometer range: more than $90 \%$ of the detected particles were 
smaller than $100 \mathrm{~nm}$. When processing a pre-treated surface, particulate emission even tends to increase, and the share of ultrafine particles below $100 \mathrm{~nm}$ clearly increases.

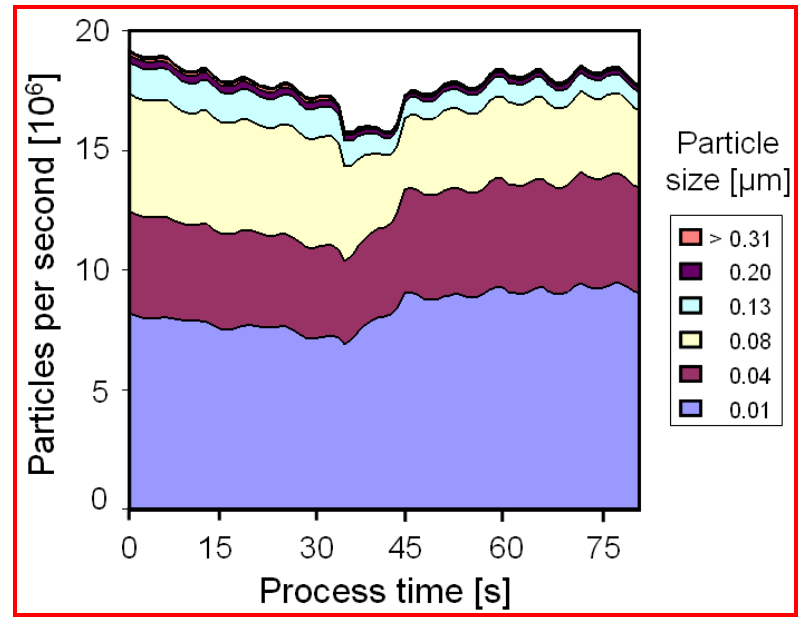

Fig. 1 Particulate emission during fs laser ablation of Y-TZP, focusing $800 \mu \mathrm{J}$ pulses onto the sample surface in air, processing a polished surface twice within 80 seconds.

One sample of nanoparticles from ultrashort-pulsed laser ablation of yttria-stabilized tetragonal zirconia is shown in Fig. 2. We have analyzed these nanoparticles using a field-emission environmental scanning electron microscope (FEG-ESEM) demonstrating by energy-dispersive X-ray spectroscopy (EDX) that the particles contain a fraction of yttria. This is in accordance with current knowledge on nanoparticle ablation using ultrashort pulses, assuming that at least a part of the generated particles are ablated stoichiometrically [8].

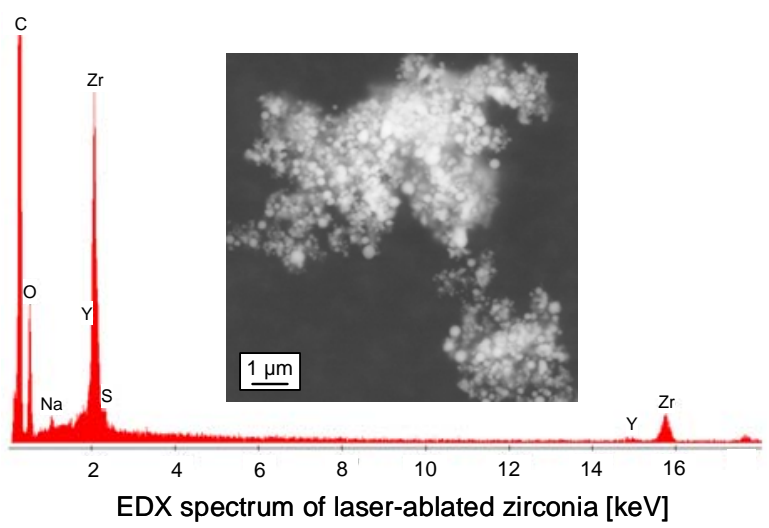

Fig. 2 FEG-ESEM analysis of nanoparticles generated by fs laser ablation of yttria-stabilized tetragonal zirconia in air.

After ablation from the zirconia surface, the zirconia nanoparticles (in micromachining terms, called “debris”) agglomerate quickly in air, re-deposit on the ablation surface, and - as they have the same morphology as the laserablated surface - attach back tightly to the original surface. This is a hindrance to precise and clean processing of zirconia in air especially when processing takes place slowly or in small areas and when the ablation products are not removed efficiently. It is known from literature that the velocity of laser-ablated matter ejected from the material decreases exponentially from the workpiece surface. Even for typical initial particle velocities in the range of several $1000 \mathrm{~m} / \mathrm{s}$, speeds are close to zero when particles have reached a distance of several millimeters from the surface [9]. Liquid nanoparticles and atomic clusters are the dominant fraction of ablated material [10] and are generally ejected later than ions and single atoms and remain closer to the ablated surface [11]. As a consequence, the emitted material settles at the sample surface close to the place of ablation due to gravitation, once it has reached a critical size due to agglomeration of air-borne nanoparticles.

Fig. 3 shows a sample area that has been processed with $10 \mu \mathrm{J}$ femtosecond laser pulses with a large pulse-topulse and line-to-line overlap in air. The first sample was processed in the focus plane and is completely covered with particulate debris that cannot be wiped away from the surface but is fixed tightly. In the second example, the focal plane was lifted upwards, allowing debris to be directly removed by the plasma pressure during the process.
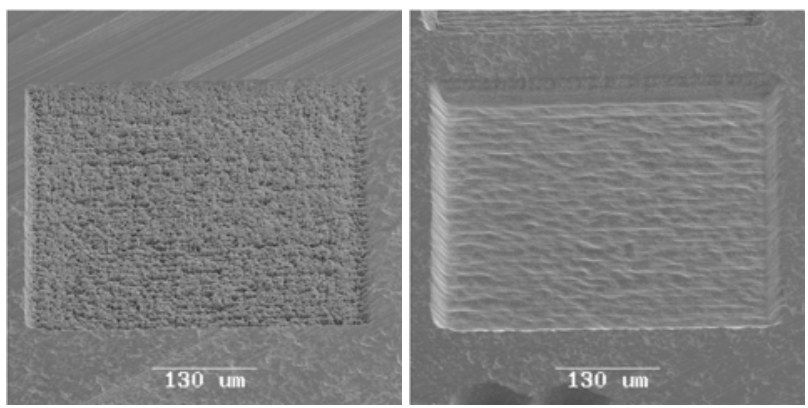

Fig. 3 Planar processing of Y-TZP using $10 \mu \mathrm{J}$ pulses at 80 $\mathrm{mm} / \mathrm{min}$ and a $5 \mu \mathrm{m}$ line pitch, focused to the sample surface (left) and $300 \mu \mathrm{m}$ above the sample surface (right).

\section{Laser micromachining through liquid layers}

However, for high pulse energies and large pulse and line overlaps, debris cannot be removed any more by varying process parameters like the focus position.

The use of a thin water layer, produced by constantly spraying water onto the sample surface during the whole time of the process, has shown to carry debris away from the processed area efficiently. A comparable improvement of femtosecond laser ablation by using a water layer has been demonstrated before at the example of silicon, showing even a significant inrease of ablation rates, which was partly due to the efficient removal of debris [12].

For the experiments, the water was applied by a commercial airbrushing set (“AB-350") at about 2 bar to disperse a constant flow of about $2 \mathrm{ml} / \mathrm{h}$ on the sample surface. Laser-generated cavities filled up completely, while the rest of the surface was wetted homogeneously.

Fig. 4 and Fig. 5 demonstrate the effect for different laser pulse energies. Each figure shows two ablated planes that were processed with identical laser and machining parameters except for the application of a water layer in the second case. This shows that especially for deep planar ablation of bulk material, a liquid layer is extremely helpful.

While the expected depth for a planar ablation with $100 \mu \mathrm{J}$ pulses and a feed rate of $80 \mathrm{~mm} / \mathrm{min}$ should be more than $200 \mu \mathrm{m}$, the first sample in Fig. 4 gives the impression of not being ablated at all. Wetting the surface with a thin water layer results in good edge qualities of the generated cavity. The thickness of the water layer increased 
significantly during the process, limiting the achievable precision and surface quality on the ground. However, the efficiency of the ablation process was still very good and much better than in air.
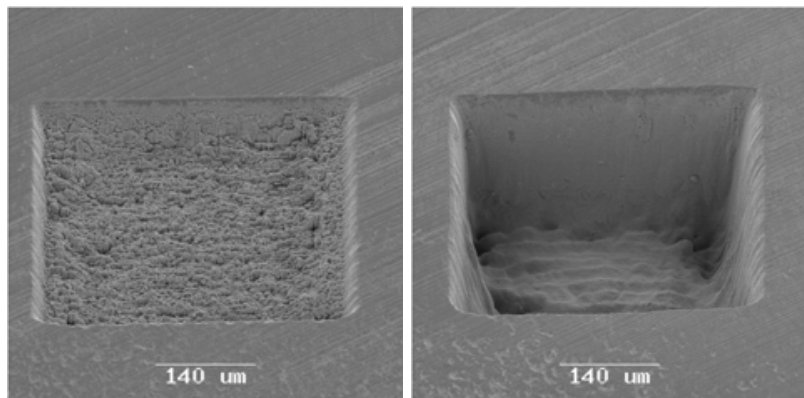

Fig. 4 Planar processing of Y-TZP using $20 \mu \mathrm{J}$ pulses at $80 \mathrm{~mm} / \mathrm{min}$ and a $5 \mu \mathrm{m}$ line pitch, focused on the sample surface in air (left) and through a thin water layer (right).
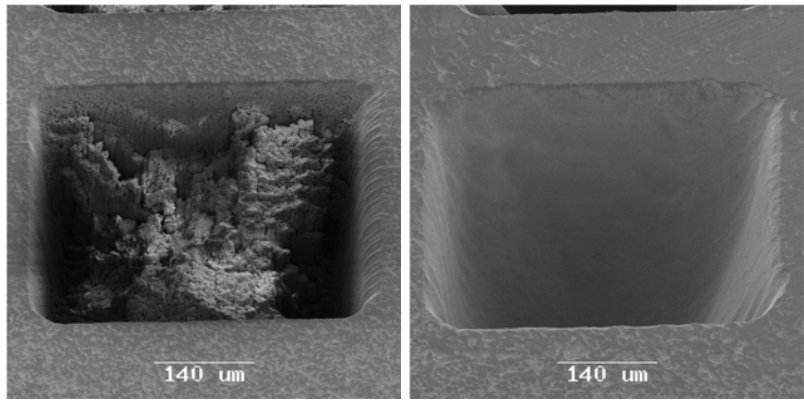

Fig. 5 Planar processing of Y-TZP using $100 \mu \mathrm{J}$ pulses at $80 \mathrm{~mm} / \mathrm{min}$ and a $5 \mu \mathrm{m}$ line pitch, focused on the sample surface in air (left) and through a thin water layer (right).

For cutting applications that involve only linear structures instead of planes, the same effect has been observed. Fig. 6 shows a groove in a zirconia sample that was cut using $80 \mu \mathrm{J}$ fs laser pulses in air and under a water layer.
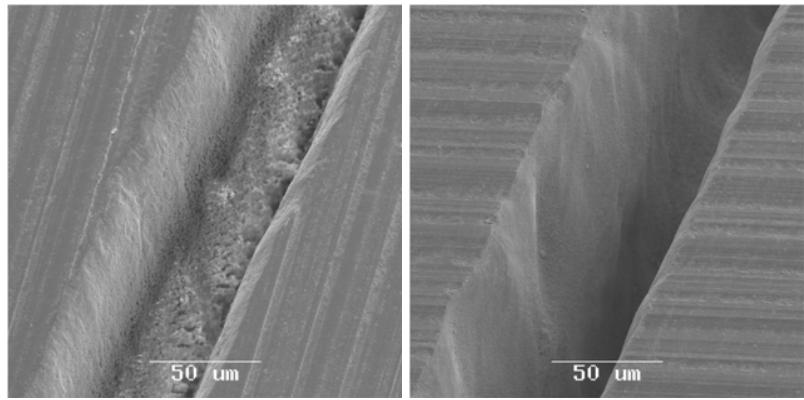

Fig. 6 Debris effect when cutting Y-TZP with $80 \mu \mathrm{J}$ pulses in air (left) and through a thin water layer (right).

Other liquids than water can also be applied to the surface and have a comparable effect. Laser-generated microstructures in Y-TZP have been generated in a vessel containing different amounts of acetone to investigate ablation through different acetone layers in comparison to air. After the experiment, the acetone, which is known to stabilize laser-ablated nanoparticles in a colloidal state, was collected to analyze the generated particle dispersions, and the sample was weighed to compare the amount of ablation.

Fig. 7 shows that the quality of the structured surface improved significantly when using the liquid layer. In this experiment, the suppression of debris deposition by a small layer of $280 \mu \mathrm{m}$ did not only clearly increase the quality of the pattern, but even increased the measured amount of ablated material.
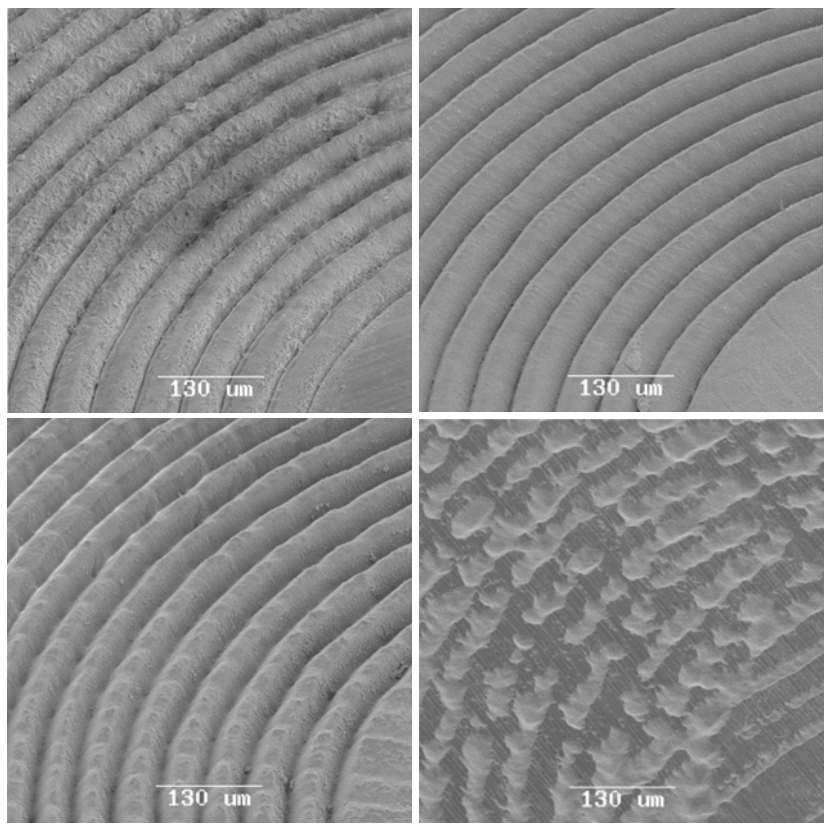

Fig. 7 Ablation of Y-TZP using $280 \mu \mathrm{J}$ pulses, focused on the sample surface in air (upper left) and through an acetone layer of $280 \mu \mathrm{m}$ (upper right), $750 \mu \mathrm{m}$ (lower left), and $1.65 \mathrm{~mm}$ (lower right).

\section{Relevance for nanoparticle generation}

When ablating tetragonal zirconia in air and through thin layers of acetone, the ablated masses are comparable, indicating that the liquid does not involve significant loss of pulse energy due to absorption. Fig. 8 shows the dependence of nanoparticle ablation rates on the thickness of the acetone layer.

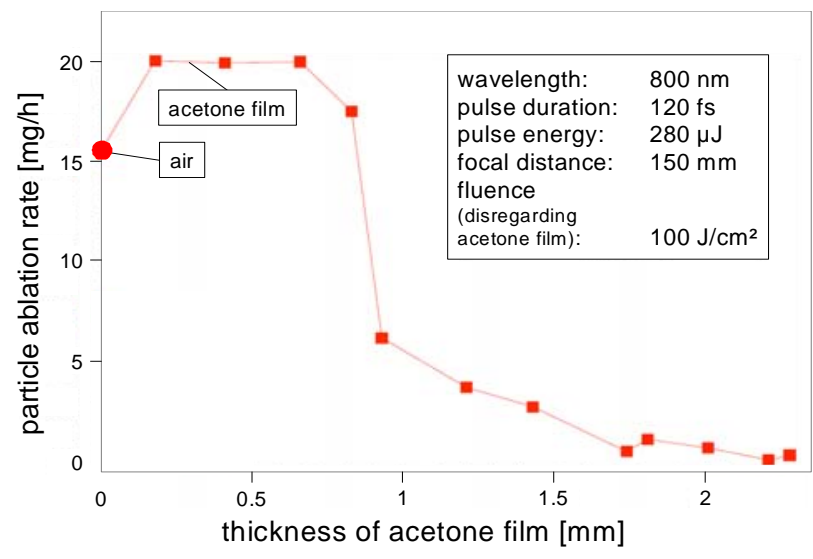

Fig. 8 fs laser ablation rates for yttria-stabilized zirconia in air (first value) and through a layer of acetone (0.2 - $2.3 \mathrm{~mm})$.

An analysis of the nanoparticle dispersions gathered from these processes was carried out using dynamic light scattering. Fig. 9 summarizes the dependence of the hydrodynamic diameter of the generated zirconia particles on the height of the acetone layer in which they were collected. The results show that while micromachining quality and ablation efficiency are increasing, the hydrodynamic size of 
the generated particles also increases significantly (indicating larger primary particles or agglomerates).

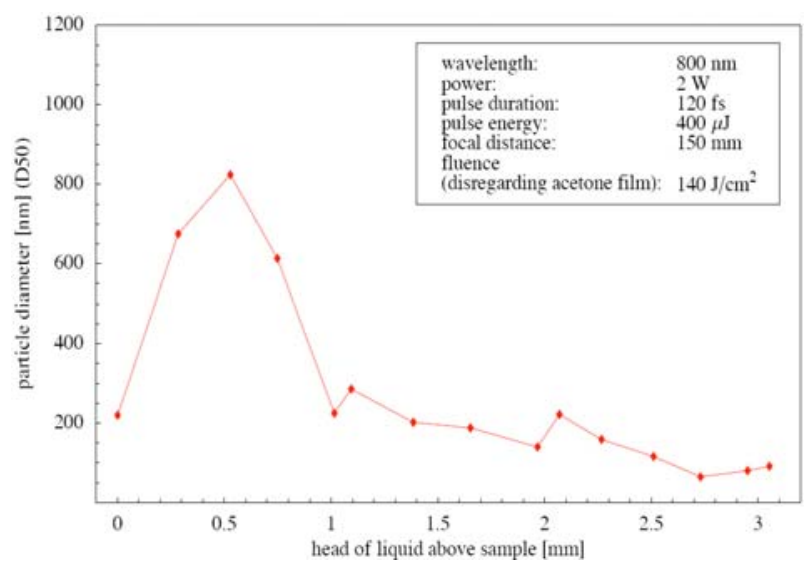

Fig. 9 Hydrodynamic diameter of zirconia nanoparticles generated by fs laser ablation in acetone.

On the other hand, crystal structure analysis of tetragonal zirconia nanoparticles from ultrashort-pulsed laser ablation provides important findings regarding unique properties: X-ray diffraction measurements have proved that that these particles maintain the tetragonal phase, which is shown in Fig. 10. The analysis was performed at a nanoparticle dispersion sample from high-power picosecond laser ablation that provided enough material for an analysis.

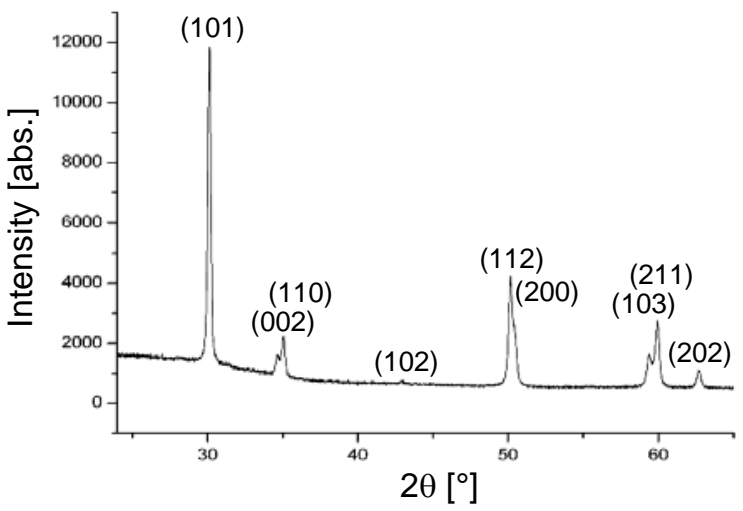

Fig. 10 Analysis of ultrashort-pulsed-laser-ablated tetragonal zirconia (from picosecond laser ablation) by x-ray diffraction, corresponding to spacegroup P42/nmc: indicating a tetragonal $\mathrm{ZrO}_{2}$ phase (courtesy of Dr. M. Wiebcke, ACI, Hannover).

The result is in agreement with the chemical composition in air (see Fig. 2), showing that the $\mathrm{ZrO}_{2}$ nanoparticles are doped with $\mathrm{Y}_{2} \mathrm{O}_{3}$ without changes in morphology. The fact that zirconia, unless doped and densely sintered, is not stable in the tetragonal phase at room temperature, suggests that the particle generation process is based on liquid confinement processes in addition with high pressure [13]. Ultrashort-pulsed laser ablation therefore provides a unique opportunity to generate tetragonal zirconia nanoparticles.

\section{Conclusion}

Both application fields for fs laser ablation of tetragonal zirconia - i.e. micromachining of zirconia parts and the generation of nanoparticle dispersions with a tetragonal crystal structure - can be combined by one processing ap- proach, involving the use of liquid films on the ablated surfaces. The investigations show a significant influence of the liquid on ablation rates, quality of micromachining, and size and amount of particulate material. Remarkably, the liquid layer does not only allow collecting nanoparticulate matter and improving the quality of micromachining, but can also enhance the productivity.

Depending on the focus of interest (quality of the ablated surface, amount of ablated material, size and amount of nanoparticles dispersed in liquids), it turns out to be recommendable to cover the ceramic surface with different amounts of liquids during the laser ablation process.

\section{Acknowledgments}

The authors acknowledge financial support by the German Federal Ministry of Education and Research (BMBF) within the projects 13N8553 and 02PU2421.

\section{References}

[1] P. Weigl, A. Kasenbacher, K. Werelius: Dental Applications. In: F. Dausinger, F. Lichtner, H. Lubatschowski (eds.): Femtosecond Technology for Technical and Medical Applications, Springer Topics in Appl. Phys. 96, 167 (2004). [2] Standard ISO 13356:1997 (E). Implants for surgery - Ceramic materials based on yttria-stabilized tetragonal zirconia (Y-TZP).

[3] N. Bärsch et al.: Femtosecond laser microstructuring of hot-isostatically pressed zirconia ceramic. J. Laser Appl. 19, 2 , 107 (2007).

[4] S. Barcikowski, A. Hahn, A. Ostendorf: Rapid Nanocomposite Manufacturing - A new way of realizing multifunctional applications. Proceedings of EuroNanoForum 2007. June 2007, 51-53.

[5] T. Kosmaç et al., The effect of surface grinding and sandblasting on flexural strength and reliability of Y-TZP zirconia ceramic. Dent. Mat. 15, 426 (1999).

[6] B. I. Ardlin, Transformation-toughened zirconia for dental inlays, crowns and bridges: chemical stabil-ity and effect of low-temperature aging on flexural strength and surface structure. Dent. Mat. 18, 590 (2002)

[7] R. G. Luthardt et al., Reliability and Properties of Ground Y-TZP-Zirconia Ceramics. J. Dent. Res. 81, 487 (2002).

[8] S. Barcikowski et al.: Properties of nanoparticles generated during femtosecond laser machining in air and water. Appl.

Phys. A 87 (2007), 47-55

[9] R. Dierken: Untersuchungen zur Entstehung von Emissionen bei der Laserstrahlmaterialbearbeitung und deren Entfernung bei abtragenden Verfahren. Thesis, ErlangenNürnberg, 1996.

[10] M. E. Povarnitsyn et al.: Material decomposition mechanisms in femtosecond laser interactions with metals. Physical Review B 75 (2008) 23, 235414

[11] K. Oguri et al.: Dynamic Imaging of Femtosecond Laser Ablation Plume by Using Laser-Generated Soft X-Ray. IEEE Lasers and Electro-Optics Society, Proceedings of LEOS 2008, 864-865

[12] J. Ren, M. Kelly, L. Hesselink, Laser ablation of silicon in water with nanosecond and femtosecond pulses. Opt Lett. 30(13), 1740-1742 (2005).

[13] G. W. Yang: Laser ablation in liquids: Applications in the synthesis of nanocrystals. Progress in Materials Science 52 (2007) 648-698 
(Received: July 7, 2008, Accepted: March 18, 2009) 